Burns, K. 156

Constantinidou, F. 145

Constantinidou, F. 119

Cruice, M. 114, 131

Eggers Viberg, M. 131

Hilari, K. 156

Hilari, K. 114, 119

Horton, S. 119

Isaksen, J. 131

Klippi, A. 113, 119

Nikou, M. 145

Northcott, S. 156
Papacostas, S. 145

Penn, C. 119

Prokopiou, J. 145

Randrup-Jensen, L. 131

Raymer, A. 119

Simpson, A. 156

Sorin-Peters, R. 114

ten Kate, O. 131

Wallace, S. 119

Worrall, L. 114, 119

Zemva, N. 119

\title{
Subject Index Vol. 67, No. 3, 2015
}

Aging 145

Aphasia 119, 131, 156

Cognition 145

Communicative participation 156

Danish speech and language therapists 131

Depression 145

Education 145

Executive functioning 145

Language 145
Memory 145

Naming 145

Professional practice 119

Psychosocial factors 145

- well-being 156

Quality of life 119, 131, 145

Solution-focused brief therapy 156 Speech-language pathology/speech and language therapy 119 Survey 119 